\title{
Global controllability of a set of fractional differential equations
}

\author{
Rabha W. Ibrahim
}




\title{
GLOBAL CONTROLLABILITY OF A SET OF FRACTIONAL DIFFERENTIAL EQUATIONS
}

\author{
RABHA W. IBRAHIM \\ Received 16 January, 2010
}

\begin{abstract}
In this paper we present the problem of global controllability (GC) of a set of fractional differential equation (SFDE).
\end{abstract}

2000 Mathematics Subject Classification: 34G10; 26A33; 34A12; 42B05

Keywords: fractional calculus, control theory, set fractional differential equations

\section{INTRODUCTION}

Recently fractional differential equations have aquired many important applications in science and engineering involving different types of differential operators (see [1-3, 5, 6, 13]). In [4], the author studied the controllability of the control system

$$
\begin{gathered}
x(t)=\mu I^{\alpha} f(t, x(t), u(t)), 0<\alpha \leq 1, \\
u(t) \in \mu G(t, x(t), u(t)), t \in J:=[0, T],
\end{gathered}
$$

where $\mu \in[0,1], G(t, x(t), u(t))$ denotes a set-valued function which has a nonempty closed convex set of values in $\mathbb{R}^{n}$ and $I^{\alpha} f(t)$ is the fractional integral operator defined as follows.

Definition 1 ([7,9,11,12]). The fractional order integral of the function $f$ of order $0<\alpha \leq 1$ is defined by

$$
I_{a}^{\alpha} f(t)=\int_{a}^{t} \frac{(t-\tau)^{\alpha-1}}{\Gamma(\alpha)} f(\tau) d \tau, \quad 0<\alpha \leq 1,
$$

and $I_{0}^{\alpha} f(t)=I^{\alpha} f(t)=f(t) * \psi_{\alpha}(t)$, where $(*)$ denotes the convolution product (see $[9,11]), \psi_{\alpha}(t)=\frac{t^{\alpha-1}}{\Gamma(\alpha)}$ for $t>0$ and $\psi_{\alpha}(t)=0$ for $t \leq 0$ and $\psi_{\alpha}(t) \rightarrow \delta(t)$ (the delta function) as $\alpha \rightarrow 0$.

Definition 2 ([7, 9, 11, 12]). The fractional order derivative of the function $f$ of order $\alpha>1$ in the Riemann-Liouville sense is defined by

$$
D_{a}^{\alpha} f(t)=\frac{d}{d t} \int_{a}^{t} \frac{(t-\tau)^{-\alpha}}{\Gamma(1-\alpha)} f(\tau) d \tau=\frac{d}{d t} I_{a}^{1-\alpha} f(t) .
$$

(c) 2011 Miskolc University Press 
Remark 1. $[9,11]$ The fractional order derivative and integral of a continuous function $f$ satisfies the relation:

$$
I^{\alpha}\left(D^{\alpha} f(t)\right)=D^{\alpha}\left(I^{\alpha} f(t)\right)=f(t) .
$$

Also we have

$$
I^{\alpha} t^{\mu}=\frac{\Gamma(\mu+1)}{\Gamma(\mu+\alpha+1)} t^{\mu+\alpha},(\mu>-1) .
$$

In this work, we consider the control set of fractional differential equations (SCFDE) as follows: Let $K_{c}\left(\mathbb{R}^{n}\right)$ be the collection of all nonempty compact convex subsets of $\mathbb{R}^{n}$

where

$$
D^{\alpha} X(t)=F(t, X(t), U(t)), 0<\alpha \leq 1,
$$

$$
\begin{gathered}
\left(X(0)=0, X(t) \in K_{c}\left(\mathbb{R}^{n}\right), U(t) \in K_{c}\left(\mathbb{R}^{m}\right), t \in J:=[0, T]\right), \\
F(t, X(t), U(t)): J \times K_{c}\left(\mathbb{R}^{n}\right) \times K_{c}\left(\mathbb{R}^{m}\right) \longrightarrow K_{c}\left(\mathbb{R}^{n}\right)
\end{gathered}
$$

and let $D^{\alpha}$ be the fractional derivative of order $\alpha \in(0,1]$. Thus (1.1) reduces to the system

$$
\left(\begin{array}{c}
D^{\alpha} x_{1}(t) \\
\vdots \\
D^{\alpha} x_{n}(t)
\end{array}\right)=\left(\begin{array}{c}
f_{1}\left(t, x_{1}(t), \ldots, x_{n}(t), u_{1}(t), \ldots, u_{m}(t)\right) \\
\vdots \\
f_{n}\left(t, x_{1}(t), \ldots, x_{n}(t), u_{1}(t), \ldots, u_{m}(t)\right)
\end{array}\right) .
$$

This paper is organized as follows: in Section 2, we recall certain basic concepts and notations which are useful in next sections. In Section 3, we present the problem of global controllability (GC) for (SCFDE), and some examples of globally controllable (GC) and non - controllable SCFDE will be given. In Section 4, we present the conclusion of this paper.

\section{PRELIMINARIES}

We recall some notations and concepts presented in detail in a recent series of works of V. Lakshmikantham et al. (see [8]).

Given $A, B \in K_{c}\left(\mathbb{R}^{n}\right)$, the Hausdorff distance between $A$ and $B$ is defined by

$$
D[A, B]:=\max \left\{\sup _{a \in A} \inf _{b \in B}\|a-b\|_{\mathbb{R}^{n}}, \sup _{b \in B} \inf _{a \in A}\|b-a\|_{\mathbb{R}^{n}}\right\}
$$

where $\|\cdot\|_{\mathbb{R}^{n}}$ denotes the Euclidean norm in $\mathbb{R}^{n}$. We define the magnitude of a nonempty subset of $A$ as

$$
D\left[A, \theta^{n}\right]=\|A\|=\sup \left\{\|a\|_{\mathbb{R}^{n}}, a \in A\right\}
$$

where $\theta^{n}$ is the zero element of $\mathbb{R}^{n}$ which is regarded as a one point set. The $\|A\|=$ $D\left[A, \theta^{n}\right]$ norm in $K_{c}\left(\mathbb{R}^{n}\right)$ is finite when the supremum in (2.1) is attained with $A \in$ $K_{c}\left(\mathbb{R}^{n}\right)$. The Hausdorff metric satisfies the following properties.

$$
D[A+C, B+C]=D[A, B] \text { and } D[A, B]=D[B, A],
$$




$$
\begin{gathered}
D[\lambda A, \lambda B]=\lambda D[A, B], D[A, B] \leq D[A, C]+D[C, B], \\
D\left[A+A^{\prime}, B+B^{\prime}\right] \leq D[A, B]+D\left[A^{\prime}, B^{\prime}\right]
\end{gathered}
$$

for all $A, B, C \in K_{c}\left(\mathbb{R}^{n}\right)$ and $\lambda \in \mathbb{R}_{+}$. If $r, s \in \mathbb{R}$ and $A, B \in K_{c}\left(\mathbb{R}^{n}\right)$, then

$$
r(A+B)=r A+r B, r(s A)=(r s) A, 1 . A=A .
$$

Finally, let $A, B \in K_{c}\left(\mathbb{R}^{n}\right)$. The set $C \in K_{c}\left(\mathbb{R}^{n}\right)$ satisfying $A=B+C$ is called the Hausdorff difference (the geometric difference) of the sets $A$ and $B$ and is denoted by the symbol $A-B$.

\section{MAIN RESUltS}

In this section we consider different cases of the system

$$
D^{\alpha} X(t)=F(t, X(t), U(t)), 0<\alpha \leq 1,
$$

where $F(t, X(t), U(t)) \in C\left[J, K_{c}\left(\mathbb{R}^{n}\right), K_{c}\left(\mathbb{R}^{m}\right)\right]$ whose solution set is of the form

$$
\begin{aligned}
X(t) & =X\left(X_{0}, t, U(t)\right) \in K_{c}\left(\mathbb{R}^{n}\right),\left(X(0)=X_{0}=0\right) \\
& =X(0, t, U(t)) \\
& :=X(t, U(t)) .
\end{aligned}
$$

Definition 3. A state pair of the solution set $\left(X_{0}, X_{1}\right) \in K_{c}\left(\mathbb{R}^{n}\right)$ is controllable if after time $t_{1}$ we can find a control set $U(t) \in K_{c}\left(\mathbb{R}^{m}\right)$ such that

$$
X\left(t_{1}\right)=X\left(X_{0}, t_{1}, U\left(t_{1}\right)\right)=X_{1} \in K_{c}\left(\mathbb{R}^{n}\right) .
$$

Definition 4. The system (3.1) is called

- (GC): Globally controllable if every state pair of solution set $\left(X_{0}, X_{1}\right) \in K_{c}\left(\mathbb{R}^{n}\right)$ is controllable;

- (GR): Globally achievable if for every $X_{1} \in K_{c}\left(\mathbb{R}^{n}\right)$ we have a state pair of solution set $\left(\theta^{n}, X_{1}\right) \in K_{c}\left(\mathbb{R}^{n}\right)$ that is controllable;

- (GNC): Globally achievable to $\theta^{n} \in K_{c}\left(\mathbb{R}^{n}\right)$ if for every $X_{1} \in K_{c}\left(\mathbb{R}^{n}\right)$ we have state pair of solution set $\left(X_{1}, \theta^{n}\right) \in K_{c}\left(\mathbb{R}^{n}\right)$ that is controllable.

- (SGR): Globally achievable set after time $t$ :

$$
R_{t}\left(X_{0}\right):=\left\{X(t) \in K_{c}\left(\mathbb{R}^{n}\right) \mid \exists U(t) \in K_{c}\left(\mathbb{R}^{m}\right): X\left(X_{0}, t, U(t)\right)=X(t)\right\}
$$

and

$$
R\left(X_{0}\right):=\bigcup_{t>0} R_{t}\left(X_{0}\right) .
$$

Definition 5. A control system (3.1) is called completely controllable (CC) if $\forall X_{0}, X_{1} \in K_{c}\left(\mathbb{R}^{n}\right)$ there exists a continuous control $U(t) \in K_{c}\left(\mathbb{R}^{m}\right), t \in J=[0, T]$ such that $X(0)=X_{0}$ and $X(T)=X_{1}$.

In virtue of Definition 4, we have the following result: 
Theorem 1. System (3.1) is

(i) (GC) if for every $X_{0} \in K_{c}\left(\mathbb{R}^{n}\right)$ we have $R\left(X_{0}\right)=K_{c}\left(\mathbb{R}^{n}\right)$;

(ii) (GR) if we have $R\left(\theta^{n}\right)=K_{c}\left(\mathbb{R}^{n}\right)$;

(iii) (GNC) if for every $X_{0} \in K_{c}\left(\mathbb{R}^{n}\right)$ we have $\theta^{n} \in R\left(X_{0}\right)$.

Remark 2. From Definition 5, every (CC) is controllable.

In the following we study several kinds of systems (3.1) :

3.1. Stationary linear control set of a fractional differential equation

Let us define the fractional system

$$
D^{\alpha} X(t)=A X(t)+B U(t), 0<\alpha \leq 1,
$$

where $X(0)=X_{0}=0 \in K_{c}\left(\mathbb{R}^{n}\right), A: K_{c}\left(\mathbb{R}^{n}\right) \rightarrow K_{c}\left(\mathbb{R}^{n}\right), B: K_{c}\left(\mathbb{R}^{m}\right) \rightarrow K_{c}\left(\mathbb{R}^{n}\right)$ are operators, whose solution set is of the form

$$
\begin{aligned}
X(t) & =X(t, U(t)) \in K_{c}\left(\mathbb{R}^{n}\right) \\
& =\frac{B}{\Gamma(\alpha)} \int_{0}^{t}\left[(t-\tau)^{\alpha-1}-A\right] U(\tau) d \tau .
\end{aligned}
$$

Theorem 2. System (3.4) is (GC) if and only if $B$ is invertible.

Proof. Sufficient condition: For $t_{1}>0$, we consider

$$
L\left(t_{1}\right):=\int_{0}^{t_{1}}\left[(t-\tau)^{\alpha-1}-A\right] d \tau .
$$

Then for all $t \in J$, the control $U(t) \in K_{c}\left(\mathbb{R}^{m}\right)$ has the form

$$
U(\tau)=B^{-1} \Gamma(\alpha) L^{-1}\left(t_{1}\right) X_{1} .
$$

In virtue of Remark 1, we have

$$
X(t)=I^{\alpha}[A X(t)+B U(t)]=A I^{\alpha} X(t)+B I^{\alpha} U(t) .
$$

Then by using Laplace transform and its inverse for the fractional integral operator (see [11], p. 104), we obtain

$$
\begin{array}{r}
\mathrm{E}\{X(t)\}=\mathrm{L}\left\{A I^{\alpha} X(t)+B I^{\alpha} U(t)\right\}=A \mathrm{E}\left\{I^{\alpha} X(t)\right\}+B \mathrm{~L}\left\{I^{\alpha} U(t)\right\} \\
\Rightarrow X(s)=A \mathrm{~L}\left\{I^{\alpha} X(t)\right\}+B \mathrm{~L}\left\{I^{\alpha} U(t)\right\} \Rightarrow X(s)=A s^{-\alpha} X(s)+B s^{-\alpha} U(s) \\
\Rightarrow X(s)\left[1-A s^{-\alpha}\right]=B s^{-\alpha} U(s) \\
\Rightarrow X(s)=\frac{B s^{-\alpha}}{1-A s^{-\alpha}} U(s)=\frac{B}{s^{\alpha}-A} U(s),
\end{array}
$$

where $\mathcal{X}(s)$ and $U(s)$ are the Laplace transform of $X$ and $U$ respectively. Hence, by the inverse Laplace transform, we have

$$
X(t)=\frac{B}{\Gamma(\alpha)} \int_{0}^{t}\left[(t-\tau)^{\alpha-1}-A\right] U(\tau) d \tau .
$$


Now for $t_{1}>0$, we state that

$$
\begin{aligned}
& X\left(t_{1}\right)=\frac{B}{\Gamma(\alpha)} \int_{0}^{t_{1}}\left[\left(t_{1}-\tau\right)^{\alpha-1}-A\right] U(\tau) d \tau \\
& =\frac{B}{\Gamma(\alpha)} \int_{0}^{t_{1}}\left[\left(t_{1}-\tau\right)^{\alpha-1}-A\right]\left[B^{-1} \Gamma(\alpha) L^{-1}\left(t_{1}\right) X_{1}\right] d \tau \\
& \quad=B B^{-1}\left(\int_{0}^{t_{1}}\left[\left(t_{1}-\tau\right)^{\alpha-1}-A\right] d \tau\right) L^{-1}\left(t_{1}\right) X_{1}=X_{1} .
\end{aligned}
$$

This implies that the system (3.4) is (GC).

Necessary condition: Assume that system (3.4) is (GC). If $B$ is not invertible then the existence of the feedback control $U(t)$ implies that system (3.4) is not (GC) and this is a contradiction.

\subsection{Non stationary linear control set of fractional differential equations}

Let us define the linear system

$$
D^{\alpha} X(t)=A(t) X(t)+B(t) U(t), 0<\alpha \leq 1,
$$

where $X(0)=X_{0} \in K_{c}\left(\mathbb{R}^{n}\right), A(t): K_{c}\left(\mathbb{R}^{n}\right) \rightarrow K_{c}\left(\mathbb{R}^{n}\right), B(t): K_{c}\left(\mathbb{R}^{m}\right) \rightarrow K_{c}\left(\mathbb{R}^{n}\right)$ are operators, whose solution set is of the form $X(t)=X\left(0, X_{0}, t, U(t)\right) \in K_{c}\left(\mathbb{R}^{n}\right)$.

Theorem 3. System (3.6) is (GC) if and only if $B^{-1}(t)$ exists.

Proof. Sufficient condition: For $t_{1}>0$, we consider

$$
\Upsilon\left(t_{1}\right):=\int_{0}^{t_{1}}\left[(t-\tau)^{\alpha-1}-A(\tau)\right] d \tau .
$$

Then for all $t \in J$, the control $U(t) \in K_{c}\left(\mathbb{R}^{m}\right)$ has the form

$$
U(\tau)=B^{-1}(\tau) \Gamma(\alpha) \Upsilon^{-1}\left(t_{1}\right) X_{1} .
$$

In view of Remark 1 , we have

$$
X(t)=I^{\alpha}[A(t) X(t)+B(t) U(t)]=I^{\alpha} A(t) X(t)+I^{\alpha} B(t) U(t) .
$$

By using the Laplace transform and its inverse for the fractional integral operator (see [11], P 104), we obtain

$$
\begin{aligned}
& \mathrm{E}\{X(t)\}=\mathrm{E}\left\{I^{\alpha} A(t) X(t)+I^{\alpha} B(t) U(t)\right\} \\
& \Rightarrow X(s)=\mathrm{E}\left\{I^{\alpha} A(t) X(t)\right\}+\mathrm{E}\left\{I^{\alpha} B(t) U(t)\right\} \\
& \Rightarrow \mathcal{X}(s)=s^{-\alpha} \mathcal{A}(s) \mathcal{X}(s)+s^{-\alpha} \mathcal{B}(s) \mathcal{U}(s) \\
& \Rightarrow \mathcal{X}(s)\left[1-\mathcal{A}(s) s^{-\alpha}\right]=\mathscr{B}(s) s^{-\alpha} \mathcal{U}(s) \\
& \Rightarrow \mathcal{X}(s)=\frac{\mathscr{B}(s) s^{-\alpha}}{1-\mathcal{A}(s) s^{-\alpha}} \mathcal{U}(s)=\frac{\mathscr{B}(s)}{s^{\alpha}-\mathcal{A}(s)} U(s) \text {, }
\end{aligned}
$$


where $\mathcal{A}(s), \mathscr{B}(s), \mathcal{X}(s)$ and $\mathcal{U}(s)$ are the Laplace transform of $A, B, X$ and $U$ respectively. Hence by the inverse of Laplace transform, we have

$$
X(t)=\frac{1}{\Gamma(\alpha)} \int_{0}^{t}\left[(t-\tau)^{\alpha-1}-A(\tau)\right] B(\tau) U(\tau) d \tau .
$$

Now, for $t_{1}>0$, this yields

$$
\begin{aligned}
X\left(t_{1}\right)= & \frac{1}{\Gamma(\alpha)} \int_{0}^{t_{1}}\left[\left(t_{1}-\tau\right)^{\alpha-1}-A(\tau)\right] B(\tau) U(\tau) d \tau \\
= & \frac{B}{\Gamma(\alpha)} \int_{0}^{t_{1}} B(\tau)\left[\left(t_{1}-\tau\right)^{\alpha-1}-A(\tau)\right]\left[B^{-1}(\tau) \Gamma(\alpha) \Upsilon^{-1}\left(t_{1}\right) X_{1}\right] d \tau \\
& =\left(\int_{0}^{t_{1}}\left[\left(t_{1}-\tau\right)^{\alpha-1}-A(\tau)\right] d \tau\right) \Upsilon^{-1}\left(t_{1}\right) X_{1}=X_{1} .
\end{aligned}
$$

This implies that system (3.6) is (GC).

Necessary condition: Assume that system (3.6) is (GC). If $B(t)$ has no inverse, then we have not the feedback control $U(t)$ implies that system (3.6) is not (GC) and this is a contradiction.

\subsection{Nonlinear control set of a fractional differential equation (NLSCFDE)}

Theorem 4. Let $F$ be continuous and $\|F\| \leq M, 0<M<\infty$. Then the control system (3.1) is (CC).

Proof. The solution set of system (3.1) takes the form

$$
X(t)=I^{\alpha} F(t, X(t), U(t)),(t \in J) .
$$

By continuity of $F$, the solution $X(t)$ is continuous. We only need to find $X_{0}$ and $X_{1}$. Since

$$
X(0)=X_{0}=0,
$$

and by Definition $1, X(t)=\frac{t^{\alpha-1} * F(t)}{\Gamma(\alpha)}$, where $(*)$ is the convolution product, we have

$$
X(T)=\frac{T^{\alpha-1} * F(T)}{\Gamma(\alpha)},(F(T) \neq 0) .
$$

We can choose $X_{0}$ and $X_{1}$ such that $X_{0}=0$ and $X_{1}=X(T)$. Hence the system (3.1) is completely controllable on $J$.

Our next aim is to show that system (3.1) is (GC). For this purpose we need to the following preliminary result..

Lemma 1. Suppose that the decreasing Lyapunov-like function $V(t, X(t), U(t))$, $t \in \mathbb{R}_{+}$satisfies the followings:

(i) $V \in C\left[\mathbb{R}_{+} \times K_{c}\left(\mathbb{R}^{n}\right) \times K_{c}\left(\mathbb{R}^{m}\right), K_{c}\left(\mathbb{R}^{n}\right)\right]$,

( ii) $\left|V\left(t, X_{1}, U_{1}\right)-V\left(t, X_{2}, U_{2}\right)\right| \leq L\left(D\left[X_{1}, X_{2}\right]+D\left[U_{1}, U_{2}\right]\right), L>0$ 
(iii) $b(\|X\|) \leq V(t, X, U) \leq a(t,\|X\|)$, for $t \in \mathbb{R}_{+}, X, X_{1}, X_{2} \in K_{c}\left(\mathbb{R}^{n}\right), U, U_{1}, U_{2} \in$ $K_{c}\left(\mathbb{R}^{m}\right)$, where $b(),. a(t,.) \in \Phi=\left\{\varphi \in C\left[\mathbb{R}_{+}, \mathbb{R}_{+}\right]: \varphi(\omega)\right.$ is increasing in $\omega$ and $\varphi(\omega) \rightarrow \infty$ as $\omega \rightarrow \infty$ \} then for every $\mu>0, t_{0} \in \mathbb{R}_{+}, \exists v>0$ such that

$$
\left\|X_{0}\right\|<\mu \text { implies }\|X(t)\|<v, \forall t>t_{0} .
$$

Proof. Given $\mu>0$, we can choose $v>0$ such that $a\left(t_{0}, \mu\right)<b(v), t_{0} \in \mathbb{R}_{+}$. If (3.7) is not hold, then for $t_{0}<t_{1}$, we obtain

$$
\begin{aligned}
b(v) \leq b\left(\left\|X\left(t_{1}\right)\right\|\right) \leq & V\left(t_{1}, X\left(t_{1}\right), U\left(t_{1}\right)\right) \\
& \leq V\left(t_{0}, X\left(t_{0}\right), U\left(t_{0}\right)\right) \leq a\left(t_{0},\left\|X_{0}\right\|\right)<a\left(t_{0}, \mu\right)<b(v) .
\end{aligned}
$$

This contradiction proves (3.7).

Theorem 5. Assume that the assumptions of Lemma 1 are satisfied. Assume that $F(t, X(t), U(t)) \in C\left[\mathbb{R}_{+} \times K_{c}\left(\mathbb{R}^{n}\right) \times K_{c}\left(\mathbb{R}^{m}\right), K_{c}\left(\mathbb{R}^{n}\right)\right]$ with

$$
\|F(t, X(t), U(t))\| \leq \widetilde{L}(\|X\|+\|U\|), \widetilde{L}>0
$$

such that

$$
\|U\| \leq \frac{\Gamma(\alpha+1)}{\widetilde{L} T^{\alpha}}\left[-\left\|X_{0}\right\|-\frac{\widetilde{L} T^{\alpha}\|X\|}{\Gamma(\alpha+1)}+\left\|X_{1}\right\|\right] .
$$

In that case the system (3.1) is (GC).

Proof. The solution set of system (3.1) satisfies the following: for $t_{1} \in J$

$$
\begin{gathered}
\left\|X\left(t_{1}\right)\right\| \leq\left\|X_{0}\right\|+\int_{0}^{t_{1}} \frac{\left(t_{1}-\tau\right)^{\alpha-1}}{\Gamma(\alpha)}\|F(\tau, X(\tau), U(\tau))\| d \tau \\
\leq\left\|X_{0}\right\|+\int_{0}^{t_{1}} \frac{\left(t_{1}-\tau\right)^{\alpha-1}}{\Gamma(\alpha)} \widetilde{L}(\|X\|+\|U\|)\|d \tau \leq\| X_{0}\left\|+\frac{T^{\alpha}}{\Gamma(\alpha+1)} \widetilde{L}(\|X\|+\|U\|)\right\| \\
\leq\left\|X_{1}\right\| .
\end{gathered}
$$

Our aim is to prove $\left\|X\left(t_{1}\right)\right\|=\left\|X_{1}\right\|$. If this is not true, i.e. $\left\|X\left(t_{1}\right)\right\|<\left\|X_{1}\right\|$, then in view of Lemma 1, we have

$$
\begin{aligned}
b(v) \leq b\left(\left\|X\left(t_{1}\right)\right\|\right) \leq V\left(t_{1},\right. & \left.X\left(t_{1}\right), U\left(t_{1}\right)\right) \\
& \leq V\left(t_{0}, X_{1}, U\left(t_{1}\right)\right) \leq a\left(t_{1},\left\|X_{1}\right\|\right)<a\left(t_{1}, \mu\right)<b(v) .
\end{aligned}
$$

That means the state pair $\left(X_{0}, X_{1}\right) \in K_{c}\left(\mathbb{R}^{n}\right)$ is controllable. Hence the (NLSCFDE) (3.1) is (GC).

In the same manner as in Theorem 5, we can prove the following result

Theorem 6. Suppose that the assumptions of Lemma 1 hold. Assume that $F(t, X(t), U(t)) \in C\left[\mathbb{R}_{+} \times K_{c}\left(\mathbb{R}^{n}\right) \times K_{c}\left(\mathbb{R}^{m}\right), K_{c}\left(\mathbb{R}^{n}\right)\right]$ with

$$
\|F(t, X(t), U(t))\| \leq \widetilde{L}(\|X\|+\|U\|), \widetilde{L}>0
$$


such that $U(t):=\sigma(t) X_{1}$ and

$$
\|\sigma\| \leq \frac{\Gamma(\alpha+1)}{\left\|X_{1}\right\| \widetilde{L} T^{\alpha}}\left[-\left\|X_{0}\right\|-\frac{\widetilde{L} T^{\alpha}\|X\|}{\Gamma(\alpha+1)}+\left\|X_{1}\right\|\right],
$$

then system (3.1) is (GC).

Remark 3. In the case $m=n$, the control operator $\sigma(t)$ must chose as a real function such that

$$
|\sigma| \leq \frac{\Gamma(\alpha+1)}{\left\|X_{1}\right\| \widetilde{L} T^{\alpha}}\left[-\left\|X_{0}\right\|-\frac{\widetilde{L} T^{\alpha}\|X\|}{\Gamma(\alpha+1)}+\left\|X_{1}\right\|\right],
$$

then system (3.1) is (GC).

We present a few examples of these two cases:

Example 1. Global controllable case: The movement of the oil trace on the sea will be controllable. Because for every pair of state sets $\left(X_{0}, X_{1}\right) \in K_{c}\left(\mathbb{R}^{n}\right) \times K_{c}\left(\mathbb{R}^{n}\right), \forall t_{1}>t_{0}, \exists U(t) \in K_{c}\left(\mathbb{R}^{m}\right)$, such that $U(t)=\sigma(t) X_{1}$, (where the control operator $\sigma(t)$ satisfies (3.8)) the NLSCFDE (3.1) is (GC).

Example 2. Non controllable case: We consider the problem of transfer of an alcohol concentrations in a volatile environment. This motion of the alcohol will not be controllable, because it might move to somewhere else so we can't find any suitable feedback $U(t)=h(X(t))$.

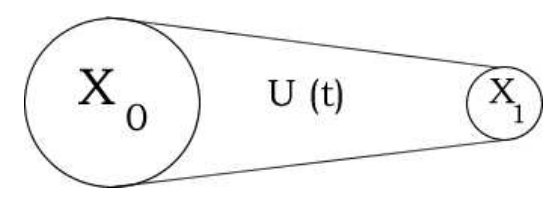

FIGURE 1. Globally controllable case

\section{CONClusion}

In this paper we used Riemann-Liouville fractional differential and integral operators for the study of the possibility of global control. We established the complete controllability of generalized systems of the form (3.1). In the case of $\alpha=1$, the system (3.1) reduces to the form

$$
D_{H} X(t)=F(t, X(t), U(t)),
$$

where $D$ is the Hukuhara derivative

$$
\begin{gathered}
\left(X(0)=X_{0}, X(t) \in K_{c}\left(\mathbb{R}^{n}\right), U(t) \in K_{c}\left(\mathbb{R}^{m}\right), t \in J:=[0, T]\right), \\
F(t, X(t), U(t)): J \times K_{c}\left(\mathbb{R}^{n}\right) \times K_{c}\left(\mathbb{R}^{m}\right) \longrightarrow K_{c}\left(\mathbb{R}^{n}\right) .
\end{gathered}
$$

This system has been studied by several authors (see $[8,10])$. 


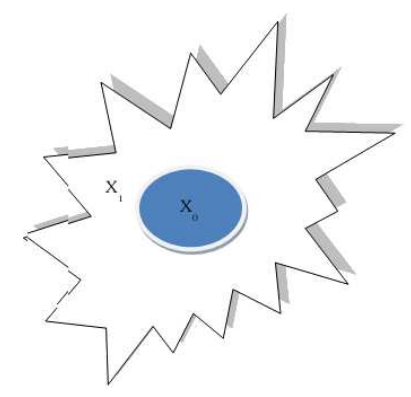

FIGURE 2. Non controllable case

\section{ACKNOWLEDGMENTS}

The author wish to thank the referee for his (or her) careful reading of this paper and for the valuable remarks which helped us to improve our presentation.

\section{REFERENCES}

[1] B. Ahmad, "Some existence results for boundary value problems of fractional semilinear evolution equations," Electron. J. Qual. Theory Differ. Equ., no. 28, p. 7, 2009.

[2] M. Benchohra and S. Hamani, "The method of upper and lower solutions and impulsive fractional differential inclusions," Nonlinear Anal. Hybrid Syst., vol. 3, no. 4, pp. 433-440, 2009.

[3] Y.-K. Chang and J. J. Nieto, "Some new existence results for fractional differential inclusions with boundary conditions," Math. Comput. Modelling, vol. 49, no. 3-4, pp. 605-609, 2009.

[4] R. W. Ibrahim, "Continuous solutions for fractional integral inclusion in locally convex topological space," Appl. Math. J. Chinese Univ. Ser. B, vol. 24, no. 2, pp. 175-183, 2009.

[5] R. W. Ibrahim, "Existence of convex and non convex local solutions for fractional differential inclusions," Electron. J. Differential Equations, no. 18, p. 13, 2009.

[6] R. W. Ibrahim and M. Darus, "Subordination and superordination for univalent solutions for fractional differential equations," J. Math. Anal. Appl., vol. 345, no. 2, pp. 871-879, 2008.

[7] V. Kiryakova, Generalized fractional calculus and applications, ser. Pitman Research Notes in Mathematics Series. Harlow/New York: Longman Scientific \& Technical/John Wiley \& Sons, Inc., 1994, vol. 301.

[8] V. Lakshmikantham, T. G. Bhaskar, and J. Vasundhara Devi, Theory of set differential equations in metric spaces. Cambridge: Cambridge Scientific Publishers, 2006.

[9] K. S. Miller and B. Ross, An introduction to the fractional calculus and fractional differential equations, ser. A Wiley-Interscience Publication. New York: John Wiley \& Sons, Inc., 1993.

[10] N. D. Phu, "On the global controllable for set differential equations," Int. J. Evol. Equ., vol. 4, no. 3, pp. 281-291, 2010.

[11] I. Podlubny, "Fractional differential equations," in An introduction to fractional derivatives, fractional differential equations, to methods of their solution and some of their applications, ser. Mathematics in Science and Engineering, vol. 198. San Diego, CA: Academic Press, Inc., 1999, pp. xxiv +340 . 
[12] S. G. Samko, A. A. Kilbas, and O. I. Marichev, "Fractional integrals and derivatives," in Theory and applications. Yverdon: Gordon and Breach Science Publishers, 1993, pp. xxxvi+976.

[13] X. Su and S. Zhang, "Solutions to boundary-value problems for nonlinear differential equations of fractional order," Electron. J. Differential Equations, no. 26, p. 15, 2009.

\section{Author's address}

\section{Rabha W. Ibrahim}

School of Mathematical Sciences, Faculty of science and Technology Universiti Kebangsaan Malaysia, Bangi 43600, Selangor Darul Ehsan, Malaysia

E-mail address: rabhaibrahimeyahoo.com 